\title{
Boyle's law and gravitational instability
}

\author{
M. Lombardi ${ }^{1}$ and G. Bertin ${ }^{2}$ \\ 1 Institüt für Astrophysik und Extraterrestrische Forschung, Universität Bonn, Auf dem Hügel 71, \\ 53121 Bonn, Germany \\ 2 Università degli Studi di Milano, Dipartimento di Fisica, via Celoria 16, 20133 Milano, Italy
}

Received 20 April 2001 / Accepted 8 June 2001

\begin{abstract}
We have re-examined the classical problem of the macroscopic equation of state for a hydrostatic isothermal self-gravitating gas cloud bounded by an external medium at constant pressure. We have obtained analytical conditions for its equilibrium and stability without imposing any specific shape and symmetry to the cloud density distribution. The equilibrium condition can be stated in the form of an upper limit to the cloud mass; this is found to be inversely proportional to the power $3 / 2$ of a form factor $\mu$ characterizing the shape of the cloud. In this respect, the spherical solution, associated with the maximum value of the form factor, $\mu=1$, turns out to correspond to the shape that is most difficult to realize. Surprisingly, the condition that defines the onset of the Bonnor instability (or gravothermal catastrophe) can be cast in the form of an upper limit to the density contrast within the cloud that is independent of the cloud shape. We have then carried out a similar analysis in the two-dimensional case of infinite cylinders, without assuming axisymmetry. The results obtained in this paper generalize well-known results available for spherical or axisymmetric cylindrical isothermal clouds that have had wide astrophysical applications, especially in the study of the interstellar medium.
\end{abstract}

Key words. equation of state - gravitation - instabilities - methods: analytical - ISM: clouds

\section{Introduction}

Consider a static isothermal gas cloud embedded in a medium characterized by an external pressure $p_{\mathrm{b}}$. Under equilibrium conditions, it is expected to satisfy the virial theorem:

$2 K+W=3 p_{\mathrm{b}} V$

where $K$ is the total internal kinetic energy associated with the thermal motions of the gas molecules and $W$ is the total gravitational energy. From the relation $K=$ $3 M c_{\mathrm{s}}^{2} / 2$, we see that

$p_{\mathrm{b}} V=M c_{\mathrm{s}}^{2}+\frac{1}{3} W<\frac{M k T}{m}$.

Here $c_{\mathrm{s}}$ is the thermal speed inside the cloud, given by $c_{\mathrm{s}}=\sqrt{k T / m}$, where $k$ is the Boltzmann constant, $T$ the cloud temperature, and $m$ the mean mass of the gas particles. Hence, the presence of gravity softens the standard equation of state $p_{\mathrm{b}} V=M k T / m$ for a gas cloud of volume $V$ (Boyle's law) into one characterized by an effective

Send offprint requests to: M. Lombardi, e-mail: lombardi@astro.uni-bonn.de temperature $T_{\text {eff }}<T$. Clearly, $T_{\text {eff }}$ depends on the size, the shape, and the temperature of the cloud. In particular, for given values of all other parameters, we could imagine density distributions for which $W$ is a large negative quantity, so that $T_{\text {eff }}<0$; these distributions would not be compatible with the assumed equilibrium conditions. On the other hand, even when $T_{\text {eff }}>0$, the equilibrium configuration might not be viable because it could be unstable.

Within spherical symmetry, this line of thought has led to the remarkable discovery (Bonnor 1956; see also Ebert $1955)$ that a thermodynamic type of instability takes place when the density contrast (between the center and the periphery of the cloud) exceeds a certain threshold value. This relatively simple conclusion has opened the way to a variety of interesting "applications", best exemplified by the interpretation of the structure of weakly collisional stellar systems (globular clusters) in terms of the onset of the so-called gravitational catastrophe (see Lynden-Bell \& Wood 1968 and following papers; in particular, see Spitzer 1987). The underlying reason for the instability can be traced to a basic property of self-gravity, of being associated with a negative specific heat. It would be conceptually interesting to study whether and how the general conclusions change for different cloud shapes. 
A study of such well-posed mathematical problem appears to be timely. Indeed, there has been enormous progress in the study of the cloudy structure of the interstellar medium (e.g., Falgarone et al. 1992; Falgarone et al. 1998; Lada et al. 1994; Alves et al. 1998). Curiously, some of the properties that are often considered as analytically useful in idealized theoretical models have been shown to be, at least in some cases, also realistic. In particular, while the possibility of non-trivial shapes for gas clouds is clearly suggested by observations of the cold interstellar medium (see, e.g., Loren 1989a; Lada et al. 1991), in some cases these observations quantitatively support a simple interpretation in terms of pure hydrostatic equilibrium (see, e.g., Alves et al. 2001). Similarly, it has long been recognized that cold clouds generally conform to the virial equilibrium conditions (e.g., see Myers 1983; Loren 1989b; Jijina et al. 1999). Furthermore, although magnetic fields and rotation are generally accepted to play a significant role in the dynamics of gas clouds (see Goodman et al. 1995), there is evidence that cloud shapes are decoupled from the presence of magnetic fields (compare the case of the Taurus cloud, Heyer et al. 1987; with that of the $\rho$ Ophiuchi cloud, Goodman et al. 1990) and that rotation can often be insignificant (e.g., Walker et al. 1993); moreover, statistical arguments applied to the distribution of measured axial ratios for cloud cores suggest that those structures are intrinsically prolate (David \& Verschueren 1987; Myers et al. 1991).

Therefore, we find it natural to address the issue of the stability of an isothermal cloud in hydrostatic equilibrium, bounded by an external medium characterized by a specified constant pressure $p_{\mathrm{b}}$, in its most general case, that is beyond the well-known framework of spherical symmetry. Since we do not consider the role of magnetic fields and, especially, non-thermal motions, we limit our discussion to the scale of cloud cores, typically a few tenths of a parsec. Still, a study on such scales is very important if we wish to understand the process of star formation. In contrast with previous analytical investigations, we then let the shape and density distribution of the cloud deviate significantly from the spherical shape. The origin of these shapes should be traced to the presence of external forces (tidal fields and/or other boundary forces). Here we will not be concerned about how a specific shape can be realized, appealing to the empirical fact that observations demonstrate that cloud cores find the way to settle in a variety of shapes. Note that filamentary structures are very common (e.g., Loren 1989a; Bally et al. 1987), and often each filament contains several distinct subcondensations, sometimes periodically spaced (Dutrey et al. 1991).

By generalizing the study by Bonnor (1956), in this paper we obtain an expression for the compressibility $\partial p_{\mathrm{b}} / \partial V$ at constant temperature and fixed shape and thus reconstruct the isothermal curves in the $\left(V, p_{\mathrm{b}}\right)$ plane. These are parameterized by a dimensionless form factor $\mu$, a quantity that depends only on the shape of the cloud, but otherwise follow the qualitative behavior characteristic of the self-gravitating isothermal sphere; in particular, no equilibrium is available above a certain value of the boundary pressure. Our analysis quantifies how such equilibrium condition depends on $\mu$ and shows that the sphere, being associated with the maximum value for the form factor $(\mu=1)$, corresponds to the shape for which equilibrium is most difficult to realize. We then proceed to address the condition for the Bonnor instability and show that it can be cast in a form that is actually independent of the cloud shape.

The paper is organized as follows. The relevant basic relations are introduced in Sect. 2. In Sect. 3 we derive the equivalent of Boyle's law for self-gravitating clouds. The implications of the results obtained for the equilibrium and stability of the cloud are clarified in Sect. 4 and are summarized in two simple conditions (Eqs. (34) and (35)). In Sect. 5 we present analogous results for (infinite) cylinders. Finally, in Sect. 6, we briefly summarize the main results obtained in this paper.

\section{Basic relations}

We summarize here the basic relations that will be used through this paper.

Let us call $\rho(\boldsymbol{x})$ the mass density distribution and $p(\boldsymbol{x})$ the (scalar) pressure of a cloud at point $\boldsymbol{x}$. If the cloud is in hydrostatic equilibrium, then

$\nabla p=-\rho \nabla \Phi$,

where $\Phi(\boldsymbol{x})$ is the Newtonian gravitational potential, described, inside the cloud, by the Poisson equation

$\nabla^{2} \Phi=4 \pi G \rho$.

Furthermore, if the cloud is isothermal we can write

$p=c_{\mathrm{s}}^{2} \rho$.

Taking $T=10 \mathrm{~K}$ and a cloud made of molecular hydrogen, we find a typical value of $c_{\mathrm{s}} \simeq 200 \mathrm{~m} \mathrm{~s}^{-1}$.

Equations (3)-(5) combined together lead to Emden's equation (Emden 1907):

$\nabla \cdot\left(\frac{\nabla \rho}{\rho}\right)=\nabla^{2} \ln \left(\rho / \rho_{0}\right)=-\frac{4 \pi G}{c_{s}^{2}} \rho$

where $\rho_{0}$ is a characteristic density (e.g., the maximum density of the cloud). A useful property of Eq. (6) that is the basis of the following analysis is the homology theorem (Chandrasekhar 1967): if $\rho(\boldsymbol{x})$ is a solution of this equation, then $\lambda^{2} \rho(\lambda \boldsymbol{x})$ is also a solution. In fact, we can write Eq. (6) in dimensionless form by introducing the variables $u=\frac{\rho}{\rho_{0}}$

and $\boldsymbol{\xi}=s \boldsymbol{x}$, where $s^{-1}$ is a suitable "Jeans length" (cf. Jeans 1929)

$s=\sqrt{\frac{4 \pi G \rho_{0}}{c_{\mathrm{s}}^{2}}}$. 
Hence $u$ is solution of the equation

$\nabla_{\xi}^{2} \ln u(\boldsymbol{\xi})=-u(\boldsymbol{\xi})$,

where $\nabla_{\xi}^{2}$ is the Laplacian with respect to the variable $\boldsymbol{\xi}$. In the following we will reserve the subscript $b$ for quantities measured at the boundary of the cloud.

\section{Boyle's law}

In this section we will derive Boyle's law for a selfgravitating cloud of arbitrary shape subject to a uniform external pressure $p_{\mathrm{b}}$. In particular, we will obtain an expression for the derivative $\partial p_{\mathrm{b}} / \partial V$ at constant temperature and fixed shape, for a cloud of given mass. A similar calculation had been performed by Bonnor (1956; see also Ebert 1955) in the case of a spherical cloud. Here we imagine that the cloud is embedded in an external medium in an arbitrary dynamical state. Our main assumption is that such an arbitrary state enforces a non-trivial geometry for the cloud, while the cloud has had time to settle into a hydrostatic equilibrium and is thus described by Eq. (9). The possibility of such non-trivial shapes is clearly suggested by observations of the cold interstellar medium (see, e.g., Loren 1989a; Lada et al. 1991), which in some cases also support the hydrostatic hypothesis (see, e.g., Alves et al. 2001).

Calculations will be carried out using the following scheme: we will first calculate the change of the cloud volume corresponding to a change of the density parameter $\rho_{0}$; then we will relate the variation of the density parameter $\rho_{0}$ to the variation of the pressure $p_{\mathrm{b}}$ at the boundary of the cloud. The shape of the cloud is taken to remain unchanged.

Since the cloud is in equilibrium with an external pressure $p_{\mathrm{b}}$, the boundary of the cloud is defined by an isodensity surface characterized by $\rho_{\mathrm{b}}=p_{\mathrm{b}} / c_{\mathrm{s}}^{2}$. When the external pressure changes, the cloud boundary remains an iso-density surface. In other words, the transformations considered involve only a change of $\rho_{0}$ (and thus of $s$ ) in $\rho(\boldsymbol{x})$, while $u(\boldsymbol{\xi})$ remains unchanged. Finally, we note that, since the boundary is an iso-density surface, $\nabla \rho$ and $\nabla_{\xi} u$ are normal to the cloud boundary.

\subsection{Perturbations at constant mass}

Suppose that the density parameter $\rho_{0}$ changes by a quantity $\delta \rho_{0}$. Then the cloud density distribution will change by

$\delta \rho(\boldsymbol{x})=\delta \rho_{0} u(s \boldsymbol{x})+\delta \rho_{0} \frac{\mathrm{d} s}{\mathrm{~d} \rho_{0}} \rho_{0} \nabla_{\xi} u(s \boldsymbol{x}) \cdot \boldsymbol{x}$.

As a result, the mass inside a given volume $V$ will change by

$\delta M(V)=\int_{V} \delta \rho(\boldsymbol{x}) \mathrm{d} V$.
From Eq. (10) we obtain

$$
\begin{aligned}
\delta M(V)= & \delta \rho_{0} \int_{V} u(s \boldsymbol{x}) \mathrm{d} V \\
& +\delta \rho_{0} \frac{\mathrm{d} s}{\mathrm{~d} \rho_{0}} \rho_{0} \int_{V} \nabla_{\xi} u(s \boldsymbol{x}) \cdot \boldsymbol{x} \mathrm{d} V .
\end{aligned}
$$

The first term on the r.h.s. of the previous equation is simply $\delta \rho_{0} M(V) / \rho_{0}$. For the second term, we can apply the general relation $\nabla(f \boldsymbol{v})=\nabla f \cdot \boldsymbol{v}+f \nabla \cdot \boldsymbol{v}$. Thus we obtain

$$
\begin{aligned}
\delta M(V)= & \frac{\delta \rho_{0} M(V)}{\rho_{0}}+\delta \rho_{0} \frac{\mathrm{d} s}{\mathrm{~d} \rho_{0}} \frac{\rho_{0}}{s} \int_{V}[\nabla \cdot(u(s \boldsymbol{x}) \boldsymbol{x}) \\
& -u(s \boldsymbol{x}) \nabla \cdot \boldsymbol{x}] \mathrm{d} V \\
= & \frac{\delta \rho_{0} M(V)}{\rho_{0}}+\delta \rho_{0} \frac{\mathrm{d} \ln s}{\mathrm{~d} \rho_{0}}\left[\oint_{\partial V} \rho_{0} u(s \boldsymbol{x}) \boldsymbol{x} \cdot \boldsymbol{n} \mathrm{d} S\right. \\
& \left.-3 \int_{V} \rho_{0} u(s \boldsymbol{x}) \mathrm{d} V\right],
\end{aligned}
$$

where we have used the Gauss theorem ( $\partial V$ is the boundary of $V, \boldsymbol{n}$ is the unit vector associated with the oriented surface element). Since $\rho$ is constant on $\partial V$, we find

$$
\begin{aligned}
\delta M(V) & =\frac{\delta \rho_{0} M(V)}{\rho_{0}}+\frac{\delta \rho_{0}}{2 \rho_{0}}\left[\rho_{\mathrm{b}} \int_{V} \nabla \cdot \boldsymbol{x} \mathrm{d} V-3 M(V)\right] \\
& =-\frac{\delta \rho_{0} M(V)}{2 \rho_{0}}+\frac{3 \delta \rho_{0} \rho_{\mathrm{b}} V}{2 \rho_{0}} .
\end{aligned}
$$

Therefore, for perturbations at constant cloud mass a variation $\delta \rho_{0}$ of the "central" density must be compensated for by a suitable change of volume $\delta V$. Since the change of mass due to a change of volume $\delta V$ is $\rho_{\mathrm{b}} \delta V$, we must have

$-\frac{\delta \rho_{0} M}{2 \rho_{0}}+\frac{3 \delta \rho_{0} \rho_{\mathrm{b}} V}{2 \rho_{0}}+\delta V \rho_{\mathrm{b}}=0$.

This equation controls the change of central density $\delta \rho_{0}$ when the volume of the cloud changes.

\subsection{Total mass in virial equilibrium}

For the following calculations it is useful to obtain a simple alternative expression for the total mass applicable to a self-gravitating isothermal cloud in hydrostatic equilibrium. For the purpose, we will use Eq. (6) in the integral of the mass:

$$
\begin{aligned}
M & =\int_{V} \rho(\boldsymbol{x}) \mathrm{d} V=-\frac{c_{\mathrm{s}}^{2}}{4 \pi G} \int_{V} \nabla \cdot\left(\frac{\nabla \rho}{\rho}\right) \mathrm{d} V \\
& =-\frac{c_{\mathrm{s}}^{2}}{4 \pi G} \oint_{\partial V} \frac{\nabla \rho \cdot \boldsymbol{n}}{\rho} \mathrm{d} S .
\end{aligned}
$$

This expression can be simplified by noting that $\rho$ is constant on $\partial V$ and $\nabla \rho \cdot \boldsymbol{n}=-\|\nabla \rho\|$, because the boundary is defined as an iso-density surface. [Here $\|\boldsymbol{A}\|=\sqrt{\boldsymbol{A} \cdot \boldsymbol{A}}$ denotes the norm of the vector $\boldsymbol{A}$.] Hence, we find

$M=\frac{c_{\mathrm{s}}^{2} \rho_{0} s}{4 \pi G \rho_{\mathrm{b}}} \oint_{\partial V}\left\|\nabla_{\xi} u\right\| \mathrm{d} S$.

The use of this relation is equivalent to the use of the equation for virial equilibrium. 


\subsection{Compressibility}

We now evaluate the change of the external pressure of a cloud as the cloud volume changes, i.e. the derivative $\partial p_{\mathrm{b}} / \partial V$ at constant temperature, constant mass, and fixed shape.

If we allow for changes of size, at the boundary Eq. (10) becomes

$$
\begin{aligned}
& \delta \rho_{\mathrm{b}}=\frac{\delta \rho_{0} \rho_{\mathrm{b}}}{\rho_{0}}+\rho_{0} \frac{\mathrm{d} s}{\mathrm{~d} \rho_{0}} \delta \rho_{0} \nabla_{\xi} u(s \boldsymbol{x}) \cdot \boldsymbol{x} \\
& -\frac{\rho_{0} s \delta V}{\oint_{\partial V}\left\|\nabla_{\xi} u(s \boldsymbol{x})\right\|^{-1} \mathrm{~d} S} .
\end{aligned}
$$

The last term on the right-hand-side of this equation can be explained as follows. Since the cloud can change its size, we expect a related variation of the external density $\rho_{\mathrm{b}}$. Such change can be written as $\delta \tilde{\rho}=-\|\nabla \rho(\boldsymbol{x})\| \delta x$ for each point $\boldsymbol{x}$, where $\delta x$ is the "stretch" of the boundary at $\boldsymbol{x}$ (we recall that this stretch is always parallel to $\nabla \rho$ ). We also note that $\delta \tilde{\rho}$ is the same for all points on the boundary of the cloud, because by definition this quantity can be identified with $\delta \rho_{\mathrm{b}}$ in the case where $\delta \rho_{0}=0$. Thus the volume changes by

$\delta V=\oint_{\partial V} \delta x \mathrm{~d} S=-\delta \tilde{\rho} \oint_{\partial V} \frac{\mathrm{d} S}{\|\nabla \rho\|}$.

Inverting this equation, we obtain that the change of density due to a modification of the size of the cloud is given by

$\delta \tilde{\rho}=-\frac{\delta V}{\oint_{\partial V}\|\nabla \rho\|^{-1} \mathrm{~d} S}=-\frac{\rho_{0} s \delta V}{\oint_{\partial V}\left\|\nabla_{\xi} u(s \boldsymbol{x})\right\|^{-1} \mathrm{~d} S}$,

which is the last term of Eq. (18).

The second term on the r.h.s. of Eq. (18) is constant for any point $\boldsymbol{x}$ on the boundary $\partial V$ because all the other terms in the equation are so. Thus we can average this constant term on the boundary using a convenient weighted mean:

$$
\begin{aligned}
\nabla_{\xi} u(s \boldsymbol{x}) \cdot \boldsymbol{x} & =\oint_{\partial V} \frac{\nabla_{\xi} u(s \boldsymbol{x}) \cdot \boldsymbol{x}}{\left\|\nabla_{\xi} u(s \boldsymbol{x})\right\|} \mathrm{d} S / \oint_{\partial V} \frac{\mathrm{d} S}{\left\|\nabla_{\xi} u(s \boldsymbol{x})\right\|} \\
& =-\oint_{\partial V} \boldsymbol{x} \cdot \boldsymbol{n} \mathrm{d} S / \oint_{\partial V} \frac{\mathrm{d} S}{\left\|\nabla_{\xi} u(s \boldsymbol{x})\right\|} \\
& =-\frac{3 V}{\oint_{\partial V}\left\|\nabla_{\xi} u(s \boldsymbol{x})\right\|^{-1} \mathrm{~d} S}
\end{aligned}
$$

With this device the second term is reduced to a form that is similar to that of the last term of Eq. (18).

\subsection{Generalized Boyle's law}

Let us briefly summarize the main results obtained so far. We wish to obtain an expression for $\partial \rho_{\mathrm{b}} / \partial V$ at constant temperature and mass or, equivalently, an expression for $\delta \rho_{\mathrm{b}}$ as a function of $\delta V$. Equation (18) is close to our need, but, unfortunately, it contains the auxiliary quantity $\delta \rho_{0}$. On the other hand, we can use Eq. (15) to express $\delta \rho_{0}$ in terms of $\delta V$ and thus eliminate $\delta \rho_{0}$ from Eq. (18). Following this procedure, we obtain the desired $\partial \rho_{\mathrm{b}} / \partial V$, but the resulting expression contains integrals that are, at first sight, difficult to interpret.

We now note that, with the help of Eq. (17), a key term involving an integration of $\left\|\nabla_{\xi} u\right\|$ (see Eqs. (18) and $(21))$ can actually be recognized to be proportional to $G M / V^{4 / 3}$ :

$\frac{1}{\oint_{\partial V}\left\|\nabla_{\xi} u(s \boldsymbol{x})\right\|^{-1} \mathrm{~d} S}=\left(\frac{4 \pi}{3}\right)^{1 / 3} \frac{M G \mu \rho_{\mathrm{b}}}{3 c_{\mathrm{s}}^{2} \rho_{0} s V^{4 / 3}}$.

This expression is reminiscent of what enters in the discussion of the spherical cloud by Bonnor (1956). For the purpose, we have introduced the dimensionless form factor as follows:

$$
\begin{aligned}
\mu= & 12 \pi\left(\frac{3}{4 \pi}\right)^{1 / 3} \frac{V^{4 / 3}}{S^{2}}\left(\frac{1}{S} \oint_{\partial V}\left\|\nabla_{\xi} u(s \boldsymbol{x})\right\| \mathrm{d} S\right)^{-1} \\
& \times\left(\frac{1}{S} \oint_{\partial V}\left\|\nabla_{\xi} u(s \boldsymbol{x})\right\|^{-1} \mathrm{~d} S\right)^{-1} .
\end{aligned}
$$

In this way, we have isolated the contribution from integrals of $\left\|\nabla_{\xi} u\right\|$. We postpone a description of the form factor to the next subsection, where the reason for the numerical factors adopted in its definition will become apparent.

Using relation (22) in Eq. (21) and combining it with Eqs. (18) and (15) we find

$\delta \rho_{\mathrm{b}}=-\frac{2 \rho_{\mathrm{b}}^{2} \delta V}{3 \rho_{\mathrm{b}} V-M}\left[1-\left(\frac{4 \pi}{3}\right)^{1 / 3} \frac{G \mu M^{2}}{6 c_{\mathrm{s}}^{2} \rho_{\mathrm{b}} V^{4 / 3}}\right]$.

Finally, using Eq. (5) we obtain the desired result:

$$
\left(\frac{\partial p_{\mathrm{b}}}{\partial V}\right)_{T, M}=-\frac{2 p_{\mathrm{b}}}{3 V} \frac{1-\left(\frac{4 \pi}{3}\right)^{1 / 3} \frac{G \mu M^{2}}{6 p_{\mathrm{b}} V^{4 / 3}}}{1-\frac{M c_{\mathrm{s}}^{2}}{3 p_{\mathrm{b}} V}}
$$

This expression generalizes Eq. (2.16) in the article by Bonnor (1956). Before discussing the physical interpretation of the equation just found and its consequences, the form factor $\mu$ deserves a special digression.

\subsection{Form factor}

The form factor, which plays a key role in the equilibrium and stability of the cloud, is defined in terms of integrals of $\left\|\nabla_{\xi} u\right\|$ and $\left\|\nabla_{\xi} u\right\|^{-1}$ on the boundary $\partial V$. Actually, the complex definition (23) has a simple interpretation in terms of general properties of $\mu$.

\subsection{1. $\mu$ is dimensionless}

As already noted, $\mu$ is a dimensionless quantity, which is a choice that allows us to preserve the scaling of the terms 
as found in Bonnor's (1956) derivation. In this respect, note that the term $V^{4 / 3}$ compensates for the two surface integrals in Eq. (23). As shown below, the introduction of a dimensionless quantity allows us to characterize and summarize the properties associated only with the shape of the cloud.

\subsection{2. $\mu$ is scale invariant}

It is easily verified that the form factor $\mu$ is scale invariant, i.e. it does not change under the transformation $\boldsymbol{x} \mapsto t \boldsymbol{x}$, with $t>0$. In other words, the form factor depends on the shape of the cloud but does not depend on its size. Actually, the scale invariance is directly related to the fact that $\mu$ is dimensionless and to the vectorial notation used in its definition.

\subsection{3. $\mu=1$ for a sphere}

In the case of a sphere, the form factor reduces to unity. In fact, in this case by symmetry $\left\|\nabla_{\xi} u(s \boldsymbol{x})\right\|$ is constant on the surface of the sphere, so that

$\mu=12 \pi\left(\frac{3}{4 \pi}\right)^{1 / 3} \frac{V^{4 / 3}}{S^{2}}=1$.

Here $S$ is the area of the surface of the sphere. Thus, from Eq. (25), we recover Bonnor's (1956) result for the sphere. Note that the numerical factors in the definition of $\mu$ have been chosen precisely to recover $\mu=1$ for the sphere.

\subsection{4. $\mu$ never exceeds unity}

The form factor can be thought of as the product of two terms, $\mu=\mu_{1} \mu_{2}$, with (cf. Eq. (26))

$\mu_{1}=12 \pi\left(\frac{3}{4 \pi}\right)^{1 / 3} \frac{V^{4 / 3}}{S^{2}}$

The first term, $\mu_{1}$, compares the volume $V$ of the cloud with its surface $S$. This quantity equals unity when the cloud is a sphere and is always smaller than unity in the non-spherical case. In fact, the sphere is the solid which has the largest volume at given surface (this intuitive theorem has a far from trivial proof, due to De Giorgi 1958). The second term, $\mu_{2}$, is the ratio between the generalized harmonic mean of $\left\|\nabla_{\xi} u\right\|$ on the boundary $\partial V$ and the generalized simple mean of the same quantity. The harmonic mean is always smaller than the simple mean, the two being equal only when all the values involved are constant. Thus $\mu_{2} \leq 1$ and $\mu_{2}=1$ if and only if $\left\|\nabla_{\xi} u\right\|$ is constant on $\partial V$ (this happens for the isothermal sphere). In conclusion, $\mu \leq 1$ in general and $\mu=1$ only for the sphere.

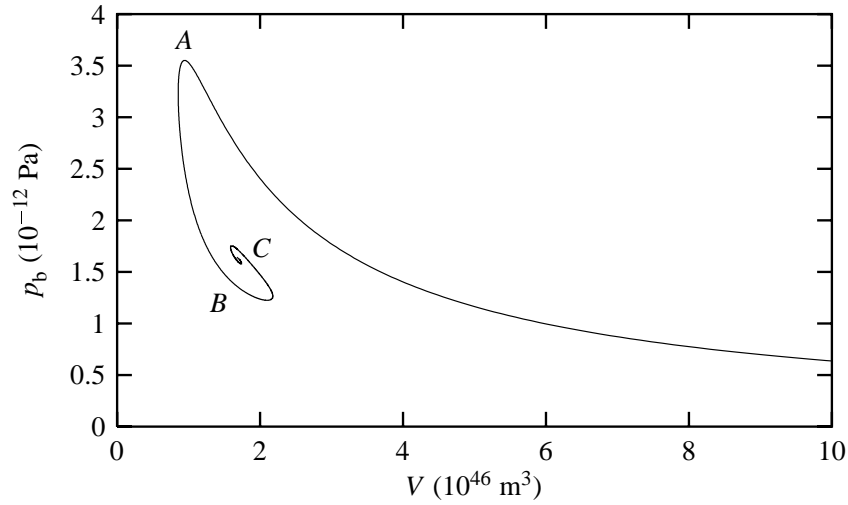

Fig. 1. The $p-V$ plot for an isothermal spherical cloud made of molecular hydrogen at $T=10 \mathrm{~K}\left(c_{\mathrm{s}} \simeq 200 \mathrm{~m} \mathrm{~s}^{-1}\right)$ with mass $M=1 M_{\odot}$. The isothermal lies on a simple $p V=$ const. curve at large volumes, but then at smaller sizes departs from it and the cloud becomes unstable. The region of stability is on the right side of point $A$ (which corresponds to the maximum of the boundary pressure $\left.p_{\mathrm{b}}\right)$. The solution spirals into the critical point $C$.

\section{The Bonnor instability}

Equation (25) allows us to discuss the Bonnor instability (often known as gravothermal catastrophe; see LyndenBell \& Wood 1968) for clouds of arbitrary shape. This instability, we recall, is due to a change in the sign of the derivative of $p_{\mathrm{b}}$ with respect to $V$. If this derivative is negative, as normally happens when gravity can be neglected, a slight decrease of the volume will produce an increase of the cloud boundary pressure, which would tend to restore the initial configuration. If, instead, this derivative is positive, then a small decrease in the volume of the cloud would correspond to a reduction of the internal boundary pressure and thus to a collapse of the cloud.

In order to illustrate the instability process in more detail, let us first refer to the spherical case. We have integrated the differential Eq. (25) numerically under the boundary condition $p_{\mathrm{b}} V=c_{\mathrm{s}}^{2} M$ for $V \rightarrow \infty$. Figure 1 shows the case of a spherical cloud of molecular hydrogen at $T=10 \mathrm{~K}\left(c_{\mathrm{s}} \simeq 200 \mathrm{~m} \mathrm{~s}^{-1}\right)$ with mass $M=1 M_{\odot}$. The plot clearly shows that the cloud follows approximately the standard law $p V=$ const at large volumes, but when sufficiently compressed it exhibits the Bonnor instability. In particular, the $p-V$ curve has a maximum and then spirals into a singular point. All points of the $p-V$ curve where the derivative is positive correspond to unstable equilibria, since a small decrease of the volume will make the cloud collapse. Bonnor has shown that actually, in the case of a spherical cloud, all points in the $p$ - $V$ plot between $A$ and the critical point correspond to unstable equilibria (below we will generalize this result to all clouds). The idea is that a spherical cloud with pressure and volume beyond $A$ will present an instability for compressions of some internal "core" (we will discuss this instability in the general case below). 


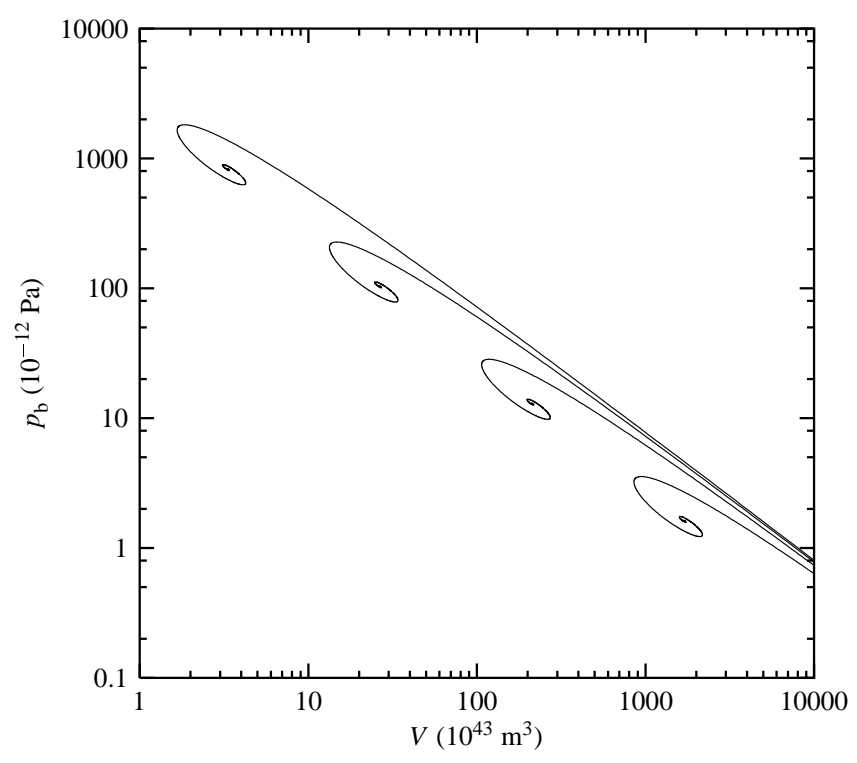

Fig. 2. The $p-V$ plot (in logarithmic coordinates) for isothermal clouds of different shapes made of molecular hydrogen at $T=10 \mathrm{~K}$ with mass $M=1 M_{\odot}$. The form factors $\mu$ are, from top left to bottom right, $0.125,0.25,0.5$, and 1 . In logarithmic coordinates, a change of $\mu$ is described by a simple translation of the isothermal curve.

In the general case of an arbitrary shape, the situation turns out to be similar. In fact, going back to Eq. (25), we can see that the form factor enters the global equation of state together with the gravitational constant $G$. In other words, a non-spherical cloud behaves like a spherical cloud with a reduced gravitational constant $G \mu$. As discussed above (see Sect. 3.5), the isothermal sphere has the largest admissible value for the form factor, $\mu=1$, and thus for the spherical shape the effects introduced by selfgravity are largest. The $G \mu$ invariance also implies that the isothermal $p$ - $V$ curves exhibit similar shapes regardless of the value of $\mu$. In Fig. 2 we have plotted the integral curves of Eq. (25) under the assumption that the cloud form factor is constant as $V$ changes. Figure 2 clearly shows that the Bonnor instability occurs at any $\mu>0$; moreover, the effect of reducing the form factor is to postpone the onset of the instability to smaller volumes. In the limit of a vanishing form factor we recover the standard law $p V=$ const. From this figure we also see that in logarithmic coordinates a change of the form factor $\mu$ simply results in a simple translation of the $p$ - $V$ curve. In fact, it is easily checked that, if we define

$$
\tilde{p}=p \mu^{3}, \quad \tilde{V}=V \mu^{-3},
$$

then Eq. (25) is transformed back into the equation appropriate for a sphere, since $\mu$ drops out. As a result, a curve relative to a form factor $\mu$ can be obtained from the curve for the sphere by multiplying the volume by $\mu^{3}$ and the pressure by $\mu^{-3}$.
Point $\mathrm{A}$ is a zero of $\partial p_{\mathrm{b}} / \partial V$ and thus satisfies

$p_{\mathrm{A}}=\left(\frac{4 \pi}{3}\right)^{1 / 3} \frac{G \mu M^{2}}{6 V_{\mathrm{A}}^{4 / 3}}$.

Using the Emden (1907) solution for the sphere, and taking advantage of the $\mu^{3}$ scaling, we can give the position of point A for arbitrary shapes:

$$
\begin{aligned}
& V_{\mathrm{A}} \simeq 0.290307\left(\frac{G \mu M}{c_{\mathrm{s}}^{2}}\right)^{3}, \\
& p_{\mathrm{A}} \simeq 1.39766 \frac{c_{\mathrm{s}}^{8}}{G^{3} \mu^{3} M^{2}} .
\end{aligned}
$$

The curves in the $p$ - $V$ plane spiral into the critical point C. This point is easily obtained by imposing that both the numerator and the denominator of Eq. (25) vanish. Then we obtain

$$
\begin{aligned}
& V_{\mathrm{C}}=\frac{\pi}{6}\left(\frac{G \mu M}{c_{\mathrm{s}}^{2}}\right)^{3} \\
& p_{\mathrm{C}}=\frac{2}{\pi} \frac{c_{\mathrm{s}}^{8}}{G^{3} \mu^{3} M^{2}}
\end{aligned}
$$

Let us now complete the discussion of the Bonnor instability for a general cloud. We have already noted that all clouds for which the derivative $\partial p_{\mathrm{b}} / \partial V$ is negative are unstable. Actually, we can show that all points in the $p-V$ curve between $\mathrm{A}$ and $\mathrm{C}$ are unstable. In fact, suppose that the cloud is in a state represented by a point B between $\mathrm{A}$ and $\mathrm{C}$, and let us consider smaller and smaller portions of the cloud with boundaries characterized by a constant pressure. Let us call $V(p)$ and $M(p)$, respectively, the volume and the mass of the part of the cloud with pressure larger than $p$. We now observe that a small compression of this part of the cloud is still described by Eq. (25) with the obvious replacements $p_{\mathrm{b}} \mapsto p, V \mapsto V(p)$, and $M \mapsto M(p)$. As we increase the value of $p$, we approach the central part of the cloud. Assuming that the maximum cloud density $\rho_{0}$ is finite, we have $M(V) \leq \rho_{0} V$, and the numerator of the last term of Eq. (25) will tend to unity because $M^{2} / V^{4 / 3} \leq \rho_{0}^{2} V^{2 / 3} \rightarrow 0$ as $V \rightarrow 0$. As a result, in the central part of the cloud the gas follows the standard law $p V=$ const., i.e. we are in the right part of the plot $p-V$. If we now move back from the central part to the boundary of the cloud represented by point $\mathrm{B}$ we must travel along the $p-V$ curve (actually, we are moving along different $p$ - $V$ curves, with respect to the reference one, because the mass $M(V)$ of the sub-cloud changes while we consider larger and larger volumes) and we will reach, at a certain boundary pressure $p_{\mathrm{a}}$, a point of type $\mathrm{A}$. In other words, we have shown that the sub-cloud characterized by $p>p_{\mathrm{a}}$ is on a point of type $\mathrm{A}$ of the $p-V$ plot. If this sub-cloud has a small negative fluctuation of its volume $V_{\mathrm{a}}=V\left(p_{\mathrm{a}}\right)$, a decrease of its boundary pressure will occur and this will lead to a further reduction of its volume. Thus we must conclude that every cloud characterized by a point in the $p-V$ plot between $\mathrm{A}$ and $\mathrm{C}$ is unstable. This is the 
generalization of the Bonnor instability to non-spherical clouds.

Point A then defines the boundary of the stability region. If the external pressure is larger than $p_{\mathrm{A}}$, no equilibrium solution exists for the cloud. This provides an upper limit for the cloud mass:

$M<M_{\text {crit }} \simeq 1.18223 \frac{c_{\mathrm{s}}^{4}}{\sqrt{G^{3} \mu^{3} p_{\text {ext }}}}$,

where $p_{\text {ext }}$ is the external pressure (that we suppose to be balanced by the cloud boundary pressure $p_{\mathrm{b}}$ ). Inserting here a typical value for the pressure of the interstellar medium, $p_{\text {ext }}=1.4 \times 10^{-13} \mathrm{~Pa}$, and using $c_{\mathrm{s}}=200 \mathrm{~m} \mathrm{~s}^{-1}$ we find $M_{\text {crit }} \simeq 4.7 \mu^{-3 / 2} M_{\odot}$.

If condition (34) is met, we can still be in the instability region if we are in the spiral part of the $p$ - $V$ curve. This happens if $p_{\mathrm{b}} V<p_{\mathrm{A}} V_{\mathrm{A}}$, and thus a necessary condition for stability, independent of $\mu$, is

$\bar{\rho}<\rho_{\text {crit }} \simeq 2.46457 \frac{p_{\text {ext }}}{c_{\mathrm{s}}^{2}}$,

where $\bar{\rho}=M / V$ is the mean density of the cloud. Inserting here the typical values used before, we find $\rho_{\text {crit }} \simeq 8.6 \times$ $10^{-18} \mathrm{~kg} \mathrm{~m}^{-3}$, corresponding to about 2600 molecules per $\mathrm{cm}^{3}$. Equation (35) can also be read in terms of a density contrast requirement $\bar{\rho}<2.46457 \rho_{\mathrm{b}}$. Equation (35) proves that the condition for the Bonnor instability can be cast in a form that is independent of the shape of the cloud. However, Bonnor's stability result for spheres, when expressed as a condition $\rho_{0} / \rho_{\mathrm{b}}<14.0$, does not hold for clouds of arbitrary shape.

Finally, we wish to emphasize that Eqs. (34) and (35) are only necessary conditions for equilibrium and stability, and not sufficient conditions. In other words, other kinds of instabilities may occur for clouds that satisfy Eqs. (34) and (35). Moreover, the presence of rotation or magnetic fields may qualitatively change these results, as suggested by recent investigations on the equilibrium of clouds in the presence of magnetic fields (see Fiege \& Pudritz 2000; Curry \& Stahler 2001).

\section{Boyle's law for (infinite) cylinders}

So far we have assumed that the cloud has finite volume. However, several observed clouds appear as filaments and can be well modeled by cylinders. The technique described above, suitably adapted, can be used also to obtain Boyle's law for (infinite) cylindrical clouds of arbitrary shape, described by a mass density of the form

$\rho\left(x_{1}, x_{2}, x_{3}\right)=\rho\left(x_{1}, x_{2}\right)$.

Note that no assumption on the function $\rho\left(x_{1}, x_{2}\right)$ has been made, and in particular we do not assume a circular section. We can now calculate the compressibility $\partial p_{\mathrm{b}} / \partial V$ following the steps taken earlier in this paper. Since the calculations are very similar to the $3 \mathrm{D}$ case, we will just outline the main differences here.
Equations (3) to (10) remain unchanged; however, since all functions are independent of $x_{3}$, it is convenient to define here $\boldsymbol{x}=\left(x_{1}, x_{2}\right), \boldsymbol{\xi}=\left(\xi_{1}, \xi_{2}\right)$, and $\nabla=\left(\partial / \partial x_{1}, \partial / \partial x_{2}\right)$. The "total" mass cannot be defined for infinite cylinders, and hence we will refer to a linear mass density $\lambda$ defined as

$\lambda=\int_{S} \rho(\boldsymbol{x}) \mathrm{d} S$,

where $S$ is the section of the cylinder.

\subsection{Perturbations at constant mass}

Using Eq. (10), which remains unchanged in the 2D case, we can write the change of "mass" $\delta \lambda(S)$ when the central density changes from $\rho_{0}$ to $\rho_{0}+\delta \rho_{0}$ assuming no variation in the cloud section $S$. The result obtained is basically the same as in Sect. 3.1 with the important difference that now $\nabla \cdot \boldsymbol{x}=2$. As a result, Eq. (14) now becomes

$$
\begin{aligned}
\delta \lambda(S) & =\frac{\delta \rho_{0} \lambda(S)}{\rho_{0}}+\frac{\delta \rho_{0}}{2 \rho_{0}}\left[\rho_{\mathrm{b}} \int_{S} \nabla \cdot \boldsymbol{x} \mathrm{d} S-2 \lambda(S)\right] \\
& =\frac{\delta \rho_{0} \rho_{\mathrm{b}} S}{\rho_{0}}
\end{aligned}
$$

Note that the two terms involving $\lambda(S)$ cancel each other. If we now allow for a change of section $\delta S$ and impose that the total "mass" $\lambda$ be constant we find the analogue of Eq. (15):

$\frac{\delta \rho_{0}}{\rho_{0}}+\frac{\delta S}{S}=0$

This equation is independent of $\rho_{\mathrm{b}}$ and simply implies $\rho_{0} \propto 1 / S$.

\subsection{Compressibility}

Equation (18) rewritten for cylinders is

$$
\begin{gathered}
\delta \rho_{\mathrm{b}}=\frac{\delta \rho_{0} \rho_{\mathrm{b}}}{\rho_{0}}+\rho_{0} \frac{\mathrm{d} s}{\mathrm{~d} \rho_{0}} \delta \rho_{0} \nabla_{\xi} u(s \boldsymbol{x}) \cdot \boldsymbol{x} \\
-\frac{\rho_{0} s \delta S}{\oint_{\partial S}\left\|\nabla_{\xi} u(s \boldsymbol{x})\right\|^{-1} \mathrm{~d} \ell} .
\end{gathered}
$$

Similarly to the 3D case, we can rewrite the second term on the r.h.s. in a more convenient way by using a suitable average. In this case we have

$$
\begin{aligned}
\nabla_{\xi} u(s \boldsymbol{x}) \cdot \boldsymbol{x} & =\oint_{\partial S} \frac{\nabla_{\xi} u(s \boldsymbol{x}) \cdot \boldsymbol{x}}{\left\|\nabla_{\xi} u(s \boldsymbol{x})\right\|} \mathrm{d} \ell / \oint_{\partial S} \frac{\mathrm{d} \ell}{\left\|\nabla_{\xi} u(s \boldsymbol{x})\right\|} \\
& =-\frac{2 S}{\oint_{\partial S}\left\|\nabla_{\xi} u(s \boldsymbol{x})\right\|^{-1} \mathrm{~d} \ell} .
\end{aligned}
$$

Inserting this result in Eq. (40) and using relation (39) we find

$\delta \rho_{\mathrm{b}}=-\frac{\delta S \rho_{\mathrm{b}}}{S}$. 
This simple result should be compared with Eq. (24), valid in the three-dimensional case. We finally find the analogue of Eq. (25),

$$
\left(\frac{\partial p_{\mathrm{b}}}{\partial S}\right)_{T, \lambda}=-\frac{p_{\mathrm{b}}}{S} .
$$

This equation states that a self-gravitating isothermal cylinder behaves like a normal gas under compressions, i.e. it follows the standard law $p_{\mathrm{b}} S=$ const. However, we should emphasize that in general the constant that appears on the r.h.s. of this expression is not equal to $\lambda c_{\mathrm{s}}^{2}$. In other words, an isothermal cylinder at temperature $T$ follows a law similar to the one of a perfect gas but at an effective temperature $T_{\text {eff }}<T$ (see Eq. (2); see also Sect. 5.3 below). The difference $T-T_{\text {eff }}$ will depend on the particular shape of the cylinder and on its linear mass density $\lambda$. Furthermore, for cylinders, since no form factor needs to be introduced, Eq. (43) can be integrated exactly without additional assumptions.

Since the cylinder follows a law similar to the standard law for perfect gases, no Bonnor instability is present in this case. Still we have to make sure that $T_{\text {eff }}>0$. From Eq. (43) we see that this is true as long as an equilibrium exists with $p_{\mathrm{b}}>0$. In the following subsection we will consider an axisymmetric cylinder in order to illustrate this point by means of a simple example.

\subsection{Axisymmetric cylinder}

The case of a circular cylinder, with density of the form $\rho(\boldsymbol{x})=\rho(r)$ dependent only on the distance $r$ from the axis, has already been the subject of several investigations (see, e.g., Ostriker 1964; Horedt 1986; Bastien et al. 1991). For an axisymmetric cylinder the exact form of the solution of Emden's equation is known (Stodołkiewicz 1963; Ostriker 1964). In our notation, the solution can be written as

$\rho(r)=\rho_{0}\left[1+\frac{(s r)^{2}}{8}\right]^{-2}$.

We can integrate this expression out to a certain radius $R$ in order to get the linear density $\lambda$ of the cylinder. On the other hand, if we know the linear density $\lambda$ and the radius $R$ of the cloud, we can obtain the central density $\rho_{0}$. A simple calculation yields

$$
\rho_{0}=\frac{\lambda}{S-\frac{G \lambda S}{2 c_{\mathrm{s}}^{2}}} .
$$

As anticipated in Eq. (39), we find $\rho_{0} \propto 1 / S$. Inserting this in Eq. (44) at $r=R$ and using Eq. (5) we finally find

$$
p_{\mathrm{b}} S=\lambda c_{\mathrm{s}}^{2}\left(1-\frac{G \lambda}{2 c_{\mathrm{s}}^{2}}\right) \text {. }
$$

This is a standard isothermal law, equivalent to Boyle's law, but characterized by an "effective" temperature

$T_{\text {eff }}=T-\frac{G \lambda m}{2 k}$.
The term $G \lambda m / 2 k$ represents the contribution from the potential energy of the cylinder, as indicated by Eq. (2). From Eq. (46) we immediately see that when the cloud linear density is larger than a critical density $\lambda_{\text {crit }}=2 c_{\mathrm{s}}^{2} / G$, the cloud cannot be in equilibrium (the required boundary pressure $p_{\mathrm{b}}$ would be negative).

\section{Conclusions}

The main results obtained in this paper can be summarized as follows.

1. The generalized Boyle's law for an isothermal selfgravitating cloud in hydrostatic equilibrium has been studied. No assumption has been made on the shape of the cloud;

2. An expression for the compressibility $\partial p_{\mathrm{b}} / \partial V$ has been obtained and, from that, two conditions for stable equilibrium have been derived;

3 . The equilibrium of the cloud has been shown to be controlled by one dimensionless form factor. The spherical shape turns out to be the one for which the effect of self-gravity is largest;

4. A simple relationship between the isothermal curves in the $p$ - $V$ plane and the form factor has been obtained;

5. The actual condition for the Bonnor instability can be written in a form that does not depend on the shape of the cloud;

6. For infinite cylinders of arbitrary shape we have shown that the Bonnor instability is suppressed.

Acknowledgements. We would like to thank João Alves for helpful discussions. Part of this work has been carried out at the Scuola Normale Superiore, Pisa, Italy.

\section{References}

Alves, J., Lada, C. J., Lada, E. A., Kenyon, S. J., \& Phelps, R. 1998, ApJ, 506, 292

Alves, J., Lada, C. J., \& Lada, E. A. 2001, Nature, 409, 159

Bally, J., Stark, A. A., Wilson, R. W., \& Langer, W. D. 1987, ApJ, 312, L45

Bastien, P., Arcoragi, J., Benz, W., Bonnell, I., \& Martel, H. 1991, ApJ, 378, 255

Bonnor, W. B. 1956, MNRAS, 116, 351

Chandrasekhar, S. 1967, An Introduction to the Study of Stellar Structure (Toronto, Dover)

Curry, C. L., \& Stahler, S. W. 2001, ApJ, in press [astro-ph/0102477]

David, M., \& Verschueren, W. 1987, A\&A, 186, 295

De Giorgi, E. 1958, Atti dell'Accademia Nazionale dei Lincei, Memorie della Classe di Scienze Matematiche Fisiche e Naturali, Sezione I (8), 5, 33

Dutrey, A., Duvert, G., Castets, A., et al. 1991, A\&A, 247, L9

Ebert, R. 1955, Zs. Ap., 217

Emden, R. 1907, Gaskugeln (Leipzig, Teubner)

Falgarone, E., Puget, J., \& Perault, M. 1992, A\&A, 257, 715 
Falgarone, E., Panis, J., Heithausen, A., et al. 1998, A\&A, 331, 669

Fiege, K., \& Pudritz, R. E. 2000, ApJ, 534, 291

Goodman, A. A., Bastien, P., Menard, F., \& Myers, P. C. 1990, ApJ, 359, 363

Goodman, A. A., Jones, T. J., Lada, E. A., \& Myers, P. C. 1995, ApJ, 448, 748

Heyer, M. H., Vrba, F. J., Snell, R .L., et al. 1987, ApJ, 321, 855

Horedt, G. P. 1986, A\&A, 160, 148

Jeans, J. H. 1929, Astronomy and Cosmogony (Cambridge University Press, Cambridge)

Jijina, J., Myers, P. C., \& Adams, F. C. 1999, ApJS, 125, 161

Lada, C. J., Lada, E.A., Clemens, D. P., \& Bally, J. 1994, ApJ, 429,694
Lada, E. A., Bally, J., \& Stark, A. A. 1991, ApJ, 368, 432

Loren, R. B. 1989a, ApJ, 338, 902

Loren, R. B. 1989b, ApJ, 338, 925

Lynden-Bell, D., \& Wood, R. 1968, MNRAS, 138, 495

Myers, P. C. 1983, ApJ, 270, 105

Myers, P. C., Fuller, G. A., Goodman, A. A., \& Benson, P. J. 1991, ApJ, 376, 561

Ostriker, J. 1964, ApJ, 140, 1056

Shu, F. H., Adams, F. C., \& Lizano, S. 1987, ARA\&A, 25, 23

Spitzer, L. 1987, Dynamical Evolution of Globular Clusters (Princeton University Press, Princeton)

Stodołkiewicz, J. S. 1963, Acta Astron., 13, 30

Walker, C. K., Carlstrom, J. E., \& Bieging, J. H. 1993, ApJ, 402,655 